\title{
LOW-FREQUENCY SOUND GENERATION BY MODULATED REPET- ITIVELY PULSED NANOSECOND PLASMA DISCHARGES
}

\author{
Olaf Bölke ${ }^{1}$, Deanna A. Lacoste ${ }^{2}$, Jonas P. Moeck ${ }^{1,3}$ \\ ${ }^{1}$ Institut für Strömungsmechanik und Technische Akustik, Technische Universität Berlin \\ 10623 Berlin, Germany, email: olaf.boelke@tu-berlin.de \\ ${ }^{2}$ Clean Combustion Research Center, King Abdullah University of Science and Technology \\ Thuwal 23955-6900, Saudi Arabia \\ ${ }^{3}$ Department of Energy and Process Engineering, Norwegian University of Science and Technology \\ 7491 Trondheim, Norway
}

\begin{abstract}
The acoustic source amplitude of low-frequency modulated spark discharges is determined experimentally. Burst and pulse-density modulation are utilized in order to generate source components at frequencies much lower than the pulse repetition frequency. Pulse sequences consist of high-voltage pulses with $10 \mathrm{~ns}$ duration, 9 to $12 \mathrm{kV}$ amplitude, and pulse repetition frequencies up to $30 \mathrm{kHz}$. The source amplitude is experimentally determined by microphone measurements in an impedance tube. Spurious components in the measured pressure signals, associated with electromagnetic noise from the high-voltage discharges, are removed by appropriate data processing. The Fourier component of the electric power at the modulation frequency is determined by phase-averaged pulse energy measurements, and the relation between electric power and sound source amplitude is revealed. The effect of pulse energy, electrode gap distance, and the number of pulses per modulation period on the initialization phase, during which HV-pulses do not generate sparks, is determined. An analytical model based on sound generation by unsteady heating is employed to estimate the acoustic source amplitude from the electrical power input; good overall agreement with the measured source amplitudes is observed. The sound generated by low-frequency modulated NRP discharges in the present work, with modulation frequencies in the range of $50-1000 \mathrm{~Hz}$, can be predicted well by assuming that the entire electrical power acts as unsteady heating over the relevant timescales.
\end{abstract}

Keywords: nanosecond discharge, low-frequency modulation, non-equilibrium plasma, sound generation

\section{Introduction}

Sound generation by gas discharges can be found in high electric fields (e.g., thunder during a storm or the crackling noise by fluctuating corona discharges in high-voltage transmission lines). Due to the resulting loss of signal quality, it is often desirable to avoid these phenomena. One of the first studies making use of this mechanism of sound generation is the "singing arc" experiment from Duddel [1], where an arc-lamp is excited to emit sound waves at frequencies in the audible frequency range. Because of the lower power consumption compared to an arc, plasma in the corona and glow regime is often used for sound generation. A review of different electric circuits for plasma sound generation is given by Mazzola et al. [2]. One aspect all methods have in common is the need of an at least partially ionized gas, which is usually provided by an electric field between two charged electrodes. However, some applications show that a flame can be used instead [3]. Advantages of plasma loudspeakers are the excellent high-frequency audio response; thus, they are used as highfidelity tweeters. Unlike traditional speakers, they have no moving parts and, therefore, a linear acoustic response. Furthermore, due to the small and robust setup, plasma speakers can be used in challenging environments. 
For an atmospheric pressure arc discharge, where the temperature increases up to $10000 \mathrm{~K}$ [4], unsteady heating is found to be the dominant sound generation mechanism. The discharge length in typical applications is short compared to the relevant acoustic wavelengths so that this sound source corresponds to a monopole, with an amplitude proportional to the rate of change of the heat energy introduced into the source per unit of time [5]. Compared to arc discharges with several tens of amperes, corona and glow plasmas are powered by low currents (less than $1 \mathrm{~A}$ ). The temperature of ions and neutrals remains almost equal to the ambient temperature [6], and the heat release rate fluctuation has a negligible effect on the sound generation in comparison to the ionic wind. The ionic wind is formed by charged species that follow the modulation of the electric field between the two electrodes. Thus, the source term is described by an acoustic dipole [5]. However, the electro-acoustical coupling mechanisms are investigated only in a few recent studies (e.g., [7]), and all mentioned studies so far focus on discharge durations of up to several milliseconds or modulated DC signals.

Recent studies have shown the potential of nanosecond repetitively pulsed (NRP) plasma for combustion applications, surface treatment, and flow control by providing significant unsteady heating and species dissociation $[8,9]$. Due to the high electron number density and the high electron temperature, interaction mechanisms of this non-equilibrium plasma with the surrounding medium can be of thermal, chemical, and gas dynamical nature. For example, non-equilibrium plasma is utilized in surface treatment applications for thin film coatings of complex carbon connections [10] and is also used for aerodynamic flow control, taking advantage of the ionic wind by surface discharges [11], between two parallel wires [12], and even in proximity of bow shocks in a supersonic airflow environment [13]. In the field of plasma-assisted combustion, nanosecond discharges have a strong impact on unsteady and transient phenomena such as combustion instabilities, ignition, and blow-off. The potential of NRP plasma in affecting the dynamics of flames, with an electrical power of less than $1 \%$ of the thermal power, is a topic of recent investigations as well as the control of combustion instabilities by low-frequency modulated plasma discharges [14-16]. An extended operational range of flames with plasma forcing is observed due to an increase in static flame stability $[17,18]$. The type of non-equilibrium plasma investigated in this context is generated by high-voltage pulses of less than $200 \mathrm{~ns}$ duration and pulse repetition frequencies of 10 to $80 \mathrm{kHz}$.

In order to improve the understanding and to distinguish the different plasma interaction mechanisms, the present study focuses on low-frequency sound generation by NRP plasma discharges. NRP discharges provide unsteady heating and species dissociation $[8,19]$. Temporal changes in heat release rate and mixture molar mass are sources of sound [20,21]. Due to the high pulse repetition rate, the propagating pressure waves were usually investigated over much shorter time scales $(<3 \mu \mathrm{s})[22$, 23 ] than relevant for typical applications, for example, in the field of combustor acoustics ( $>1 \mathrm{~ms})$. In this study, low-frequency sound waves are generated by burst and pulse-density modulation of pulse sequences with pulse repetition frequencies of $15-30 \mathrm{kHz}$, consisting of nanosecond pulses with $10 \mathrm{~ns}$ duration and voltage amplitudes up to $12 \mathrm{kV}$. Both modulation methods provide the possibility to affect the acoustic field at frequencies much lower than the pulse repetition frequency. Furthermore, the pressure amplitude is controlled by adjusting the number and/or the temporal position of the discharges in one period. In an initial study [24], it was shown that acoustic waves can be generated in the targeted frequency range by low-frequency burst modulation and that this effect can be used for control applications, such as suppressing thermoacoustic instabilities.

To assess the sound generation from modulated NRP discharges in a quantitative manner, the acoustic source amplitude is determined experimentally in an impedance tube, by means of the socalled multi-microphone method [25]. Furthermore, an analytic estimate of the source amplitude based on the measured electric power at the modulation frequency is introduced. Results are presented for different modulation frequencies, pulse repetition rates, duty cycles, and electrode gap distances. 


\section{Sound generation from compact sources in chemically reacting gases}

We consider a compact source of sound, i.e., one whose spatial extent is negligibly small compared to the acoustic wavelength. The latter is of the order $c / f$, where $c$ is the speed of sound and $f$ the oscillation frequency. In a quiescent medium, a sound source can be quantified in terms of fluctuations of the fluid expansion $\nabla \cdot \mathbf{u}$ (u is the velocity vector). The velocity divergence can be written on the basis of the mass balance:

$$
\nabla \cdot \mathbf{u}=-\frac{1}{\rho} \frac{\mathrm{d} \rho}{\mathrm{d} t}
$$

where $\rho$ is the density and $\mathrm{d} / \mathrm{d} t$ a material derivative. For an ideal gas with adiabatic species conversion, the density derivative can be written as [26]

$$
\frac{1}{\rho} \frac{\mathrm{d} \rho}{\mathrm{d} t}=\frac{1}{\gamma p} \frac{\mathrm{d} p}{\mathrm{~d} t}-\sigma
$$

where $p$ is the pressure and $\gamma$ the ratio of specific heats; diffusive effects have been omitted. The quantity $\sigma$, which is sometimes called thermicity [26], is given by

$$
\sigma=\frac{1}{\rho} \sum_{s, r}\left(\frac{M}{M_{s}}-\frac{h_{s}}{c_{p} T}\right) v_{s r} M_{s} r_{r}
$$

and mediates the transfer of chemical energy to mechanical energy through species conversion. Here, $M_{s}$ is the molar mass of species $s$ and $h_{s}$ its enthalpy, $r_{r}$ is the rate of the $r$ th reaction, $v_{s r}$ are stoichiometric coefficients, and $M$ and $c_{p}$ are mixture molar mass and heat capacity at constant pressure; $T$ is the temperature. The first term in the brackets in Eq. (3) is related to a change in particle density, and the second corresponds to the heat release rate. Both are known as sources of sound in low-Mach-number reactive flows [21].

Now for a compact acoustic source, we can take $\sigma$ to be essentially of delta support. Substituting then (3) and (2) in Eq. (1) and integrating over the arbitrarily small source volume yields

$$
\int_{\partial V} \mathbf{u} \cdot \mathrm{d} \mathbf{A}=\int_{V} \sigma \mathrm{d} V,
$$

where the pressure integral has been neglected in view of the compactness of the source. In a ducted configuration, in which only plane acoustic waves propagate (Fig. 1), we can thus write

$$
\left(u_{\mathrm{ds}}-u_{\mathrm{us}}\right) A=\Sigma \text {, }
$$

where $u$ is the axial velocity component, 'us' and 'ds' refer to the states immediately upstream and downstream of the source, and $A$ is the cross-sectional area of the duct; $\Sigma$ is the spatially integrated thermicity. Since Eq. (5) is linear in all quantities, it holds in the same form in the frequency domain.

The one-dimensional acoustic field in a duct has two degrees of freedom at every axial location; in frequency domain, these can be given by, for example, acoustic pressure and velocity phasors $\hat{p}$ and $\hat{u}$. Alternatively, the acoustic state can be expressed in terms of the complex amplitudes of the downstream and upstream traveling waves, $\hat{f}$ and $\hat{g}$, which are related to the primitive variables through [27]

$$
\hat{p}=\hat{f}+\hat{g}, \quad \rho_{0} c_{0} \hat{u}=\hat{f}-\hat{g},
$$

where $\rho_{0}$ and $c_{0}$ are mean density and mean speed of sound, respectively. By successively adding and subtracting $\hat{p}_{\text {ds }} /\left(\rho_{0} c_{0}\right)$ and $\hat{p}_{\text {us }} /\left(\rho_{0} c_{0}\right)$ to Eq. (5), and noting that the two terms are equal because the Mach number vanishes and the temperature does not change significantly, we obtain the two relations

$$
\hat{f}_{\mathrm{ds}}-\hat{f}_{\mathrm{us}}=\frac{\rho_{0} c_{0}}{2 A} \Sigma, \quad \hat{g}_{\mathrm{us}}-\hat{g}_{\mathrm{ds}}=\frac{\rho_{0} c_{0}}{2 A} \Sigma
$$




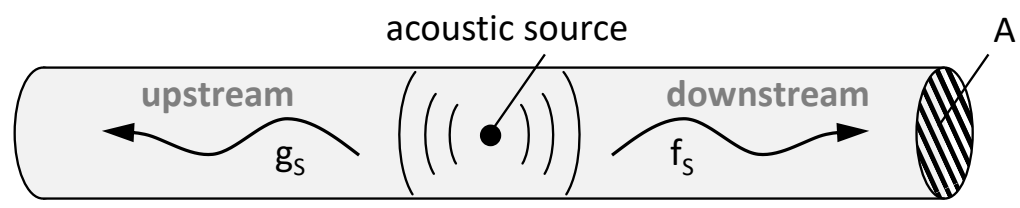

Figure 1: Sketch of duct with acoustically compact source and source-related wave amplitudes $f_{S}, g_{S}$; assuming ideal anechoic terminations

The differences in the wave amplitudes across the source domain quantify the acoustic source in terms of pressure waves emitted upstream and downstream. In the following, these source terms are denoted by $\hat{f}_{s}=\hat{f}_{\mathrm{ds}}-\hat{f}_{\mathrm{us}}$ and $\hat{g}_{s}=\hat{g}_{\text {us }}-\hat{g}_{\mathrm{ds}}$.

In combustion phenomena with air as oxidizer, the first term in (3), which is related to changes in the mixture molar mass or, in other words, to an increase or decrease in mole number, is insignificant compared to the heat release rate (because of the dilution by essentially inert nitrogen) [21]. The situation for NRP discharges is less clear. Part of the discharge energy (20\% [28], 35\% [29]) is spent on the dissociation of oxygen molecules, which would contribute to the first term in (3). Dissociation of $\mathrm{N}_{2}$ can be considered negligible [28]. The remaining energy accounts for two effects: ultra-fast gas heating, approximately $25 \mathrm{~ns}$ after the discharge, and the excitation of the vibrational modes of molecular oxygen and nitrogen [30].

Non-equilibrium discharges can provide a significant amount of heating [8, 29, 30]. Due to the short duration of the discharge and the spatial inhomogeneity of the plasma, only a few experiments on the efficiency of fast heating processes were conducted in high reduced electric fields $(E / N>80 \mathrm{Td})$. Recent investigations on ultra-fast heating provided by non-equilibrium discharges showed that between $(25 \pm 10) \%$ and $(75 \pm 25) \%[23,31]$ of the total energy input in nanosecond air discharges is transferred to gas heating, between 20 up to $3000 \mathrm{~ns}$ after the discharge. However, these experimental investigations were focused on much shorter time scales than relevant for the acoustic investigations presented in this work $(\approx 1 \mathrm{~ms})$. During the post-discharge period $(>1 \mu \mathrm{s})$, quenching processes and vibrational-translational relaxation provide additional gas heating [30, 32, 33]. We can thus hypothesize that, for timescales of $1 \mathrm{~ms}$ or larger, which are relevant for the generation of acoustic waves with frequencies up to $1 \mathrm{kHz}$, most of the discharge energy is converted into heat. If, accordingly, the change in mixture molar mass is neglected in (3), the source amplitudes can be written as

$$
\hat{f}_{s}=\hat{g}_{s}=\frac{\gamma-1}{2 A c_{0}} \hat{Q}
$$

where $\hat{Q}$ is the frequency domain quantity associated with the spatially integrated heat release rate:

$$
Q=\int_{V} \sum_{s, r} h_{s} v_{s r} M_{s} r_{r} \mathrm{~d} V .
$$

It is shown in Section 5.3 that this is in good agreement with the experimental data acquired in the present work.

\section{Low-frequency modulation of NRP discharges}

Non-equilibrium plasma in this work is generated by NRP discharges with pulse repetition frequencies of up to $30 \mathrm{kHz}$. In order to generate acoustic waves at frequencies much lower than the pulse repetition frequency, the high-frequency pulse train is modulated at frequencies in the range of 80 to $1000 \mathrm{~Hz}$. The most simple way to generate pressure oscillations with short-duration pulses at frequencies much lower than the inverse pulse length is to generate discharges with a constant pulse repetition frequency equal to the desired frequency. However, this method is quite static in terms of 

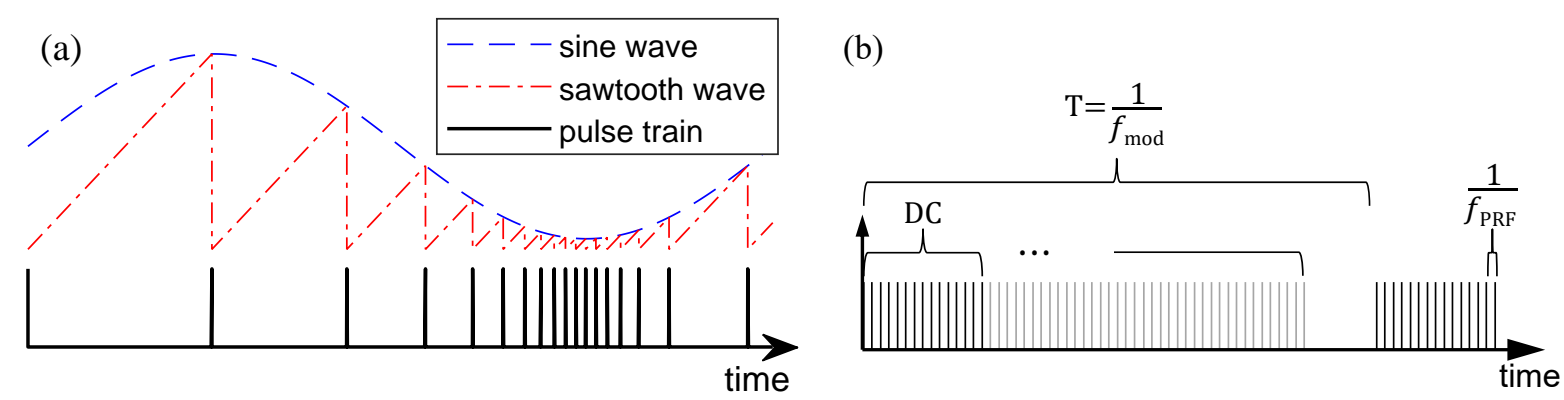

Figure 2: Illustration of the modulation methods: generation of pulse train by pulse-density modulation (a) and burst-modulated signal, including different duty cycles (b).

pulse number and pulse density, thus power, which can be delivered as acoustic excitation; moreover, the power that can be provided in this way is generally low. More flexible methods are the pulse-density modulation (PDM) and the pulse-width modulation (PWM), where the latter represents a special case of the PDM. Further modulation methods are pulse-position modulation (PPM) and the burst modulation (BM). Limitations in the choice of the modulation method are given by the highvoltage pulse generator (FID Technologies, FPG 10-30NM10) which is utilized for the investigations in this work. Only high-voltage pulses with a constant pulse width of $10 \mathrm{~ns}$ as well as constant energy per pulse can be produced. Due to these limitations, investigations presented here focus on PDM and BM.

Figure 2(a) illustrates the principle of PDM. The dashed line represents a sine wave, which is to be encoded in pulses (solid line). To determine the instant of each pulse, a sawtooth signal with constant slope is generated (dash-dotted). Every time the saw-tooth hits the sine wave, a pulse is set, and the sawtooth starts again. The number of pulses per period is determined by a combination of the offset and the amplitude of the sine wave as well as the slope of the sawtooth. Burst-modulated signals are generated from a high-frequency pulse train with a constant pulse repetition frequency that is gated by a rectangular waveform. The modulation frequency and the number of pulses are adjusted by the period and the duty cycle of the rectangular waveform (Fig. 2(b)).

To estimate the power amplitude at the modulation frequency, the modulated pulse sequence is expressed as a Fourier series:

$$
w(t)=w_{0}+\sum_{n=1}^{\infty}\left|w_{n}\right| \cos \left(n \Omega t+\arg w_{n}\right), \quad \text { with } \quad w_{n}=\frac{2}{T} \int_{0}^{T} w(t) \mathrm{e}^{-\mathrm{i} \Omega t_{n}} \mathrm{~d} t .
$$

Here, $\Omega=2 \pi f_{\text {mod }}$ and $f_{\text {mod }}$ is the modulation frequency. For the target frequency range of 100$1000 \mathrm{~Hz}$, the modulation period is much longer than the width of the high-voltage pulses, the latter being of the order of 10-20 ns. The high-voltage pulse train can then be represented as a sequence of Delta functions, with each peak weighted by the corresponding pulse energy. Therefore, the periodic sequence can be written as the sum of each pulse at position $t_{i}$ with $0 \leq t_{i}<T=f_{\bmod }^{-1}$ and energy $e_{n}$ :

$$
w(t)=\sum_{n=1}^{N} e_{n} \delta\left(t-t_{n}\right),
$$

where the sum runs over all pulses in one modulation period. The pulse-train amplitude at the modulation frequency, $\hat{W}$, is then given by

$$
\hat{W}=\left|w_{1}\right|=\left|\frac{2}{T} \sum_{n=1}^{N} e_{n} \mathrm{e}^{-\mathrm{i} \Omega t_{n}}\right| .
$$

Assuming identical contributions of all pulses $\left(e_{n} \equiv e\right)$, one obtains a theoretical estimate of the low-frequency amplitude depending on the pulse position and number of pulses through Eq. (12). 


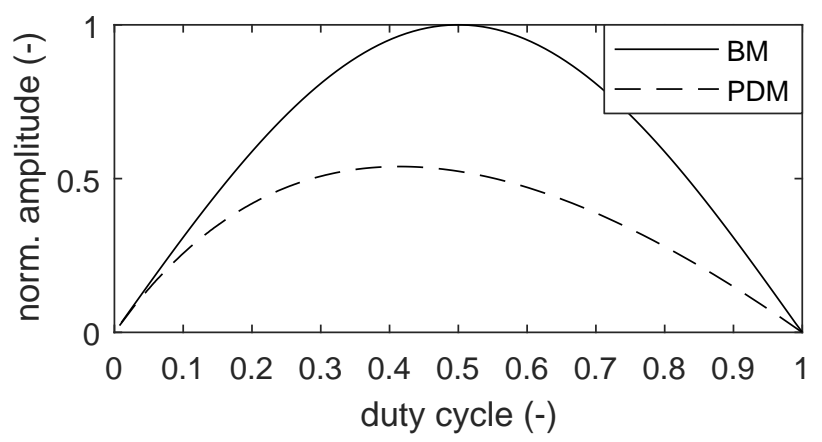

Figure 3: Calculated amplitudes of burst (solid line) and pulse-density modulated signals (dashed line) for the full duty cycle range

In order to generate signals with different source amplitude at the modulation frequency, the number of pulses per period (the duty cycle) is adjusted in this work. Under the assumption of constant pulse energy with a constant fraction of energy converted into acoustic power, the effect of maximum pulse number and pulse position on the amplitude at the modulation frequency can be calculated with Eq. (12). Figure 3 shows the normalized amplitude as a function of the duty cycle. A duty cycle of unity corresponds to the maximum number of pulses per period. For both modulation methods the maximum number of pulses is defined by the ratio of the maximum pulse repetition frequency and the modulation frequency: $N_{\max }=f_{\mathrm{PRF}} / f_{\text {mod }}$. Actuation signals with zero or $N_{\max }$ pulses evidently lead to a zero amplitude at the modulation frequency. The latter is equal to a pulse sequence with equidistant pulses. For burst-modulated signals, the duty-cycle dependence of the measured source amplitudes is proportional to $\sin (\pi \mathrm{DC})$, and the maximum amplitude is achieved at a duty cycle of $50 \%$. Due to the properties of the modulation method, the maximum amplitude of pulse-density modulated signals is slightly shifted to fewer pulses, equal to a duty cycle of $41 \%$. The theoretical maximum achieved with PDM reaches $54 \%$ of the burst-modulated amplitude only (Fig. 3).

\section{Experimental determination of sound generated by modulated NRP dis- charges}

The modulated NRP discharges are acoustically investigated by axially distributed microphones in an impedance tube. The tube has a total length of $2.35 \mathrm{~m}$ and a diameter of $40 \mathrm{~mm}$ (Fig. 4). Five 1/4-in. condenser microphones (G.R.A.S. 40BP with preamplifier 26AC) are flush mounted each upstream (us) and downstream (ds) of the acoustic source (S). The microphone signals are low-pass filtered and amplified with a custom-made signal conditioner and sampled at $65536 \mathrm{~Hz}$ with a data acquisition board (National Instruments cRIO 9074 with NI 9220 module). At half-length of the impedance tube, two tungsten electrodes (2.4 $\mathrm{mm}$ diameter) are mounted in a pin-to-pin configuration; thus, the discharge region is located in the center of the tube (Fig. 5). In order to avoid undesired discharges between the electrodes and the duct, the duct-segment is made of perspex. The electrodes are sharpened with an opening angle of $75^{\circ}$, which is a compromise between durability and ignitability. Figures 5(b) and (c) show pictures of the discharge region with non-modulated spark discharges $\left(f_{\mathrm{PRF}}=30 \mathrm{kHz}\right)$ at two different exposure times of the camera [(b) 1/2 sec and (c) 1/500 sec]. A compact discharge region between both tips of the electrodes can be observed. Acoustical foam (Basotect ${ }^{\circledR}$ ) is placed over a length of $45 \mathrm{~cm}$ at both ends inside the impedance tube in order to reduce modal resonances and improve the measurement accuracy. Plasma discharges produce species, which contaminate the air in the duct. In order to ensure consistent air composition, small flushing channels with a diameter of $5 \mathrm{~mm}$ carry a weak purging flow (less than $0.2 \mathrm{~m} / \mathrm{s}$, at a diameter of $40 \mathrm{~mm}$ ), which continuously removes the produced species.

Low-frequency trigger sequences of one period, for modulation frequencies between 50 and 


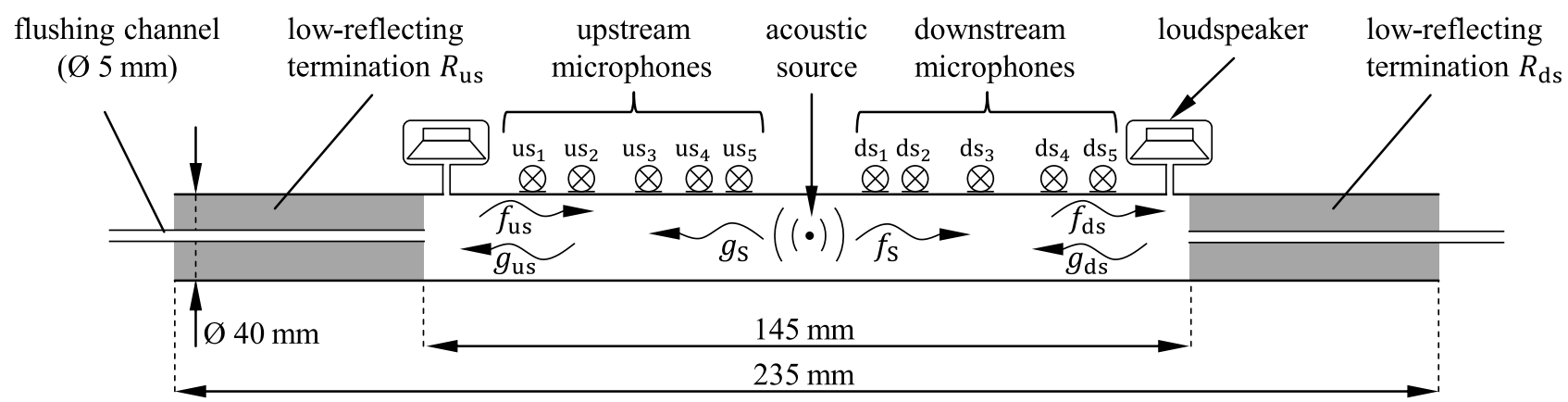

Figure 4: Experimental arrangement for acoustic source measurements: impedance tube set-up with five microphones, one loudspeaker upstream and downstream of an acoustic source, and low-reflecting terminations with flushing channels.
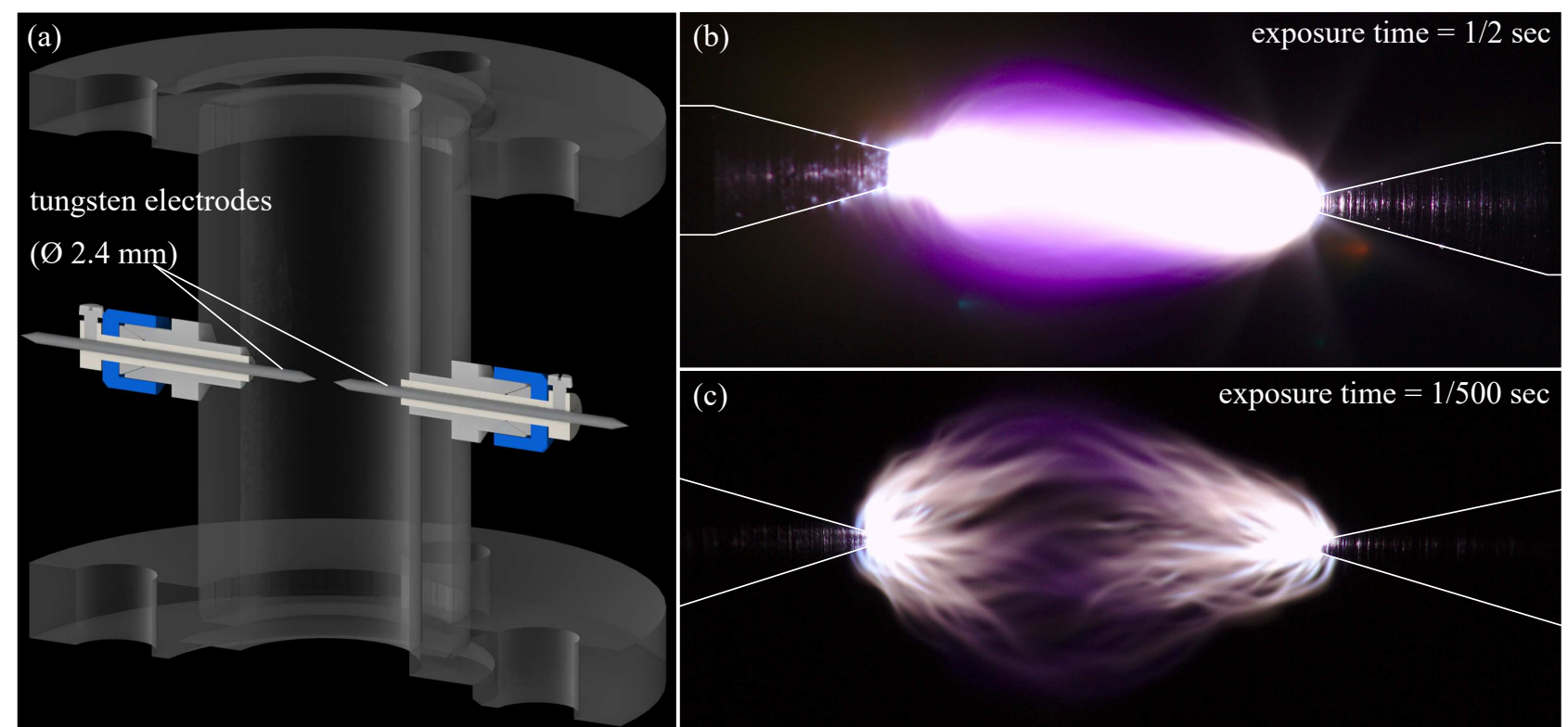

Figure 5: Acoustic source segment. Perspex duct section with two tungsten electrodes (a) and picture of the discharge region, gap distance $4 \mathrm{~mm}$, non-modulated spark discharges with $f_{\mathrm{PRF}}=30 \mathrm{kHz}$; images taken with an exposure time of $1 / 2 \mathrm{sec}(\mathrm{b})$ and $1 / 500 \mathrm{sec}(\mathrm{c})$.

$1000 \mathrm{~Hz}$, are generated by both methods described in Section 3. Theses trigger sequences are sent periodically via a digital 5V/TTL module (NI 9401) connected to a National Instruments data acquisition board (cRIO 9074) to the trigger port of the high-voltage generator. Due to the limited sampling rate of $40 \mathrm{MHz}$, small variations in the modulation frequencies occur. This error becomes largest for the highest modulation frequency but is bounded by $1.2 \times 10^{-3} \%$.

\subsection{Identification of the acoustic field with the multi-microphone method}

For frequencies below $1.84 \times c_{0} /(\pi d) \approx 5 \mathrm{kHz}$, only plane waves propagate in the tube $(d$ is the tube diameter, Fig. 4) [34]. The pressure field in the frequency domain is then given by

$$
\hat{p}(x, \omega)=\hat{f}(\omega) \mathrm{e}^{-\mathrm{i} k x}+\hat{g}(\omega) \mathrm{e}^{\mathrm{i} k x},
$$

where $\hat{p}(x, \omega)$ is the acoustic pressure at axial position $x$ and angular frequency $\omega=2 \pi f$, and $k=\omega / c_{0}$ is the acoustic wavenumber. $\hat{f}$ and $\hat{g}$ correspond to the complex amplitudes of the downstream and upstream traveling acoustic waves, respectively. The two wave amplitudes $\hat{f}$ and $\hat{g}$ can be determined 
as follows. Equation (13) is written for the $N_{\text {mic }}$ measurement locations:

$$
\left(\begin{array}{c}
\hat{p}_{1} \\
\hat{p}_{2} \\
\vdots \\
\hat{p}_{N_{\text {mic }}}
\end{array}\right)=\left(\begin{array}{cc}
\mathrm{e}^{-\mathrm{i} k x_{1}} & \mathrm{e}^{\mathrm{i} k x_{1}} \\
\mathrm{e}^{-\mathrm{i} k x_{2}} & \mathrm{e}^{\mathrm{i} k x_{2}} \\
\vdots & \vdots \\
\mathrm{e}^{-\mathrm{i} k x_{N_{\text {mic }}}} & \mathrm{e}^{\mathrm{i} k x_{N_{\text {mic }}}}
\end{array}\right)\left(\begin{array}{l}
\hat{f} \\
\hat{g}
\end{array}\right),
$$

For a given frequency $\omega$, this system is inverted in a in a least-squares sense to obtain $\hat{f}$ and $\hat{g}$.

The whole acoustic system, sketched in Fig. 4, can be characterized by the back and forth traveling waves $\hat{f}_{\mathrm{us}}, \hat{f}_{\mathrm{ds}}, \hat{g}_{\mathrm{us}}, \hat{g}_{\mathrm{ds}}$ upstream and downstream of the acoustic source. Theses quantities are linked by the scattering matrix $\mathbf{S}$ and the two source components $\hat{f}_{s}$ and $\hat{g}_{s}$ [25]:

$$
\left(\begin{array}{l}
\hat{f}_{\mathrm{ds}} \\
\hat{g}_{\mathrm{us}}
\end{array}\right)=\left(\begin{array}{ll}
S_{11} & S_{12} \\
S_{21} & S_{22}
\end{array}\right)\left(\begin{array}{l}
\hat{f}_{\mathrm{us}} \\
\hat{g}_{\mathrm{ds}}
\end{array}\right)+\left(\begin{array}{l}
\hat{f}_{s} \\
\hat{g}_{s}
\end{array}\right)
$$

The scattering matrix $\mathbf{S}$ describes reflection and transmission of the incident waves $f_{\text {us }}$ and $g_{\text {ds }}$ at and through the acoustic element. In the present investigation, the acoustic element is represented by the tungsten electrodes (Fig. 5). The source components $\hat{f}_{s}$ and $\hat{g}_{s}$ are independent of the incident field, as they are generated by the modulated discharge pulse train. The scattering matrix is determined through the application of the two-source method [35]. However, $\mathbf{S}$ is found to be the identity matrix within experimental uncertainty and thus can be considered acoustically transparent with respect to the incident wave field. Given the wave amplitudes upstream and downstream of the discharge element, which are determined by inversion of Eq. (14), the source components are determined as

$$
\hat{f}_{\mathrm{s}}=\hat{f}_{\mathrm{ds}}-\hat{f}_{\mathrm{us}}, \quad \hat{g}_{\mathrm{s}}=\hat{g}_{\mathrm{us}}-\hat{g}_{\mathrm{ds}} .
$$

\subsection{Mitigation of measurement noise}

Acoustic measurement signals are always contaminated by noise, related to, for example, turbulent flow, cooling fans of electrical devices, electronically induced noise, and other surrounding sounds. Noise uncorrelated to the excitation signal can be significantly reduced in frequency domain by blockaveraging (e.g., Welch's method). Due to the short rise time and the short pulse duration of the high-voltage discharges applied in this study, strong electromagnetic disturbances propagate from the discharge. These disturbances propagate through the air and cables with almost the speed of light. Consequently, all microphones and electrical devices are affected by the electromagnetic (EM) field nearly at the same time, but due to small variations (e.g., in cable length, electrical resistance, orientation to the propagating electromagnetic wave) not necessarily in the same manner. Due to the coupling of the sound and the EM-field generation, these disturbances are correlated to the excitation signal, and thus, cannot be removed by block-averaging. In order to reduce the impact of the EM disturbances on the data acquisition system, each microphone is covered by a copper envelope including a copper mesh terminating the membrane side of the microphone, Fig. 6(b). Ten meter long cables, connecting microphones and signal amplifier, are each surrounded by a copper mesh over the entire length and electrically grounded on both sides, Fig. 6(a). In addition to that, 10 ferrites are evenly distributed along each cable to further decrease the impact of the EM-field. The noise contamination of the microphone signals was generally lowest when data acquisition board and microphone amplifier were placed as far as possible from the discharge. However, the correlated noise component was found to be still significant in some test cases.

The spurious components in the measured pressure phasors, associated with the correlated EMnoise, are removed as follows. The frequency dependent reflection coefficient $R$ relates reflected to incident wave [27]; for example, downstream of the measurement segment, we have: $\hat{g}_{\mathrm{ds}}=R_{\mathrm{ds}} \hat{f}_{\mathrm{ds}}$, and an analogous relation holds on the upstream side. Analogous to the determination of the scattering matrix, the incident waves are determined by loudspeaker excitation upstream and downstream of 


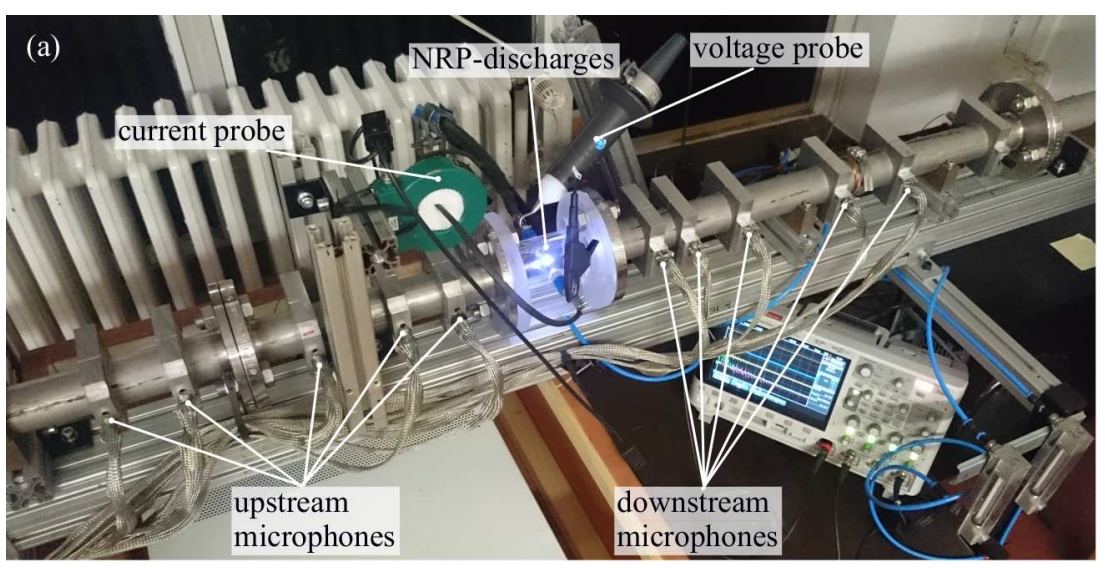

(b)

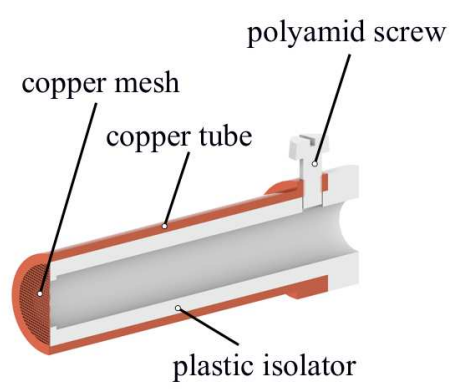

Figure 6: (a) Image of impedance tube for acoustic source measurements including voltage and current probes; microphones upstream and downstream of the acoustic source element are covered in copper mesh, including several ferrites; (b) copper envelope of microphones

the source element. Since the reflection coefficients do not change when the plasma discharges are activated, the ratio of incident to reflected wave remains the same in both conditions. The reflection coefficients are then measured without discharges (no EM-noise), and this ratio of reflected to incident wave is enforced onto the pressure phasors measured with discharges. Consider the measured, noisecontaminated vector of pressure phasors on the downstream side:

$$
\hat{\mathbf{p}}_{\mathrm{ds}, \text { meas }}=\hat{\mathbf{p}}_{\mathrm{ds}}+\hat{\mathbf{s}}
$$

where $\hat{\mathbf{p}}_{\mathrm{ds}}=\left(\begin{array}{lll}\hat{p}_{1} & \hat{p}_{2} & \ldots\end{array}\right)^{T}$ are the true pressure phasors, and $\hat{\mathbf{s}}$ is noise correlated with the excitation. The vector of true pressure phasors is then expressed by means of the reflection coefficient and the amplitude of the downstream traveling wave:

$$
\hat{\mathbf{p}}_{\mathrm{ds}}=\mathbf{a} \hat{f}_{\mathrm{ds}}, \quad \text { with } \quad \mathbf{a}=\left(\begin{array}{cc}
\mathrm{e}^{-\mathrm{i} k x_{1}} & \mathrm{e}^{\mathrm{i} k x_{1}} \\
\mathrm{e}^{-\mathrm{i} k x_{2}} & \mathrm{e}^{\mathrm{i} k x_{2}} \\
\vdots & \vdots
\end{array}\right)\left(\begin{array}{c}
1 \\
R_{\mathrm{ds}}
\end{array}\right) .
$$

With given downstream reflection coefficient, the wave amplitude $\hat{f}_{\mathrm{ds}}$ is determined from the measured pressure phasors by removing all components orthogonal to a:

$$
\hat{f}_{\mathrm{ds}}=\frac{\mathbf{a}^{H} \hat{\mathbf{p}}_{\mathrm{ds}, \text { meas }}}{|\mathbf{a}|^{2}}
$$

where $(\cdot)^{H}$ denotes the Hermitian transpose. The upstream traveling wave on the downstream side is then simply obtained from $\hat{g}_{\mathrm{ds}}=R_{\mathrm{ds}} \hat{f}_{\mathrm{ds}}$. An analogous procedure on the upstream side yields the two wave amplitudes $\hat{f}_{\text {us }}$ and $g_{\text {us }}$.

The reflection coefficients upstream and downstream of the acoustic element, $R_{\mathrm{us}}$ and $R_{\mathrm{ds}}$, are determined with loudspeaker excitation and without plasma forcing. Only for this purpose, loudspeakers (Monacor, KU-516) near both low-reflecting terminations (see Fig. 4) were mounted. These measurements are performed with the same conditions (purging flow, ambient temperature, microphone locations etc.)

\subsection{Measurement of the spark energy}

To relate the measured sound source amplitudes to the discharge power, it is important to only use the Fourier component at the modulation frequency. The electrical pulse energy was determined from electrode voltage and current, measured simultaneously with the acoustic signals. Figure 7 shows the 


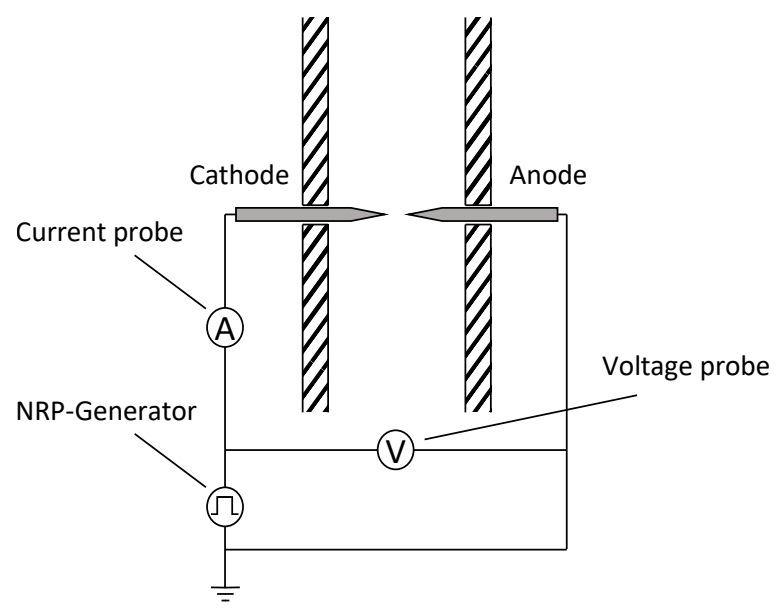

Figure 7: Experimental arrangement for electrical measurements: electrodes with current and voltage probe connected to electrical circuit with nanosecond pulse generator.

electrical circuit containing the current probe (Pearson current monitor Model 6585), the high-voltage probe (PMK 4002), and the pulse generator (FID Technologies, FPG 10-30NM10) connected to the two tungsten electrodes. The pulse energy $e_{n}$ in conjunction with the corresponding time $t_{n}$ in Eq. (12) determine the electrical power at the modulation frequency.

The local electric field between the electrodes is strongly dependent on the radius of curvature of the electrode tips. Due to the high voltage and high pulse repetition frequency, a fast erosion of the tungsten tips occurs. This strongly affects the possibility to create discharges, the breakdown voltage and the energy per pulse. In pre-tests, reproducible results could be achieved with long-time measurements of more than $30 \mathrm{~min}$. The results presented here were obtained with electrodes which are used for less than 20 min.

To determine the correct energy per pulse, the time delay (skew) of the voltage and the current signals has to be taken into account. The skew is calculated from low-voltage measurements without plasma discharges, whereby the air in the gap between the two tungsten electrodes is assumed to have an ideal capacity $C$ [36] with

$$
I_{\text {disp }}=C \frac{\mathrm{d} U}{\mathrm{~d} t}
$$

where $I_{\text {disp }}$ and $U$ denote the measured current and voltage signal. Therefore, the skew is determined by the time shift of the measured current and the calculated current signal by Eq. (20). In the present study, the skew is determined to approximately $2 \mathrm{~ns}$.

In the spark regime, the measured total current $I_{\text {tot }}$ is the sum of a displacement $I_{\text {disp }}$ and a conduction current $I_{\text {cond }}$ [29]. $I_{\text {disp }}$ corresponds to the charging of the capacitance formed by the electrodes, and $I_{\text {cond }}$ to the flowing electrons through the plasma [29]. In fact, the relevant part of the current, which provides ultra-fast air heating, is the conductive current. The displacement current is measured at initial pulses with an energy of less than $100 \mu \mathrm{J}$, for which no discharge occurs. Representative current and voltage signals, as well as the corresponding energy and power, are shown in Fig. 8. In the spark regime, the displacement component of the measured current is small compared to the conductive one (see Fig. 8(b)).

A peak voltage of $11 \mathrm{kV}$ and a measured total current of $55 \mathrm{~A}$ is reached, which results in a peak power of $200 \mathrm{~kW}$ and an energy per pulse of $2.6 \mathrm{~mJ}$. In the following, only the energy calculated with the conductive current is used. 

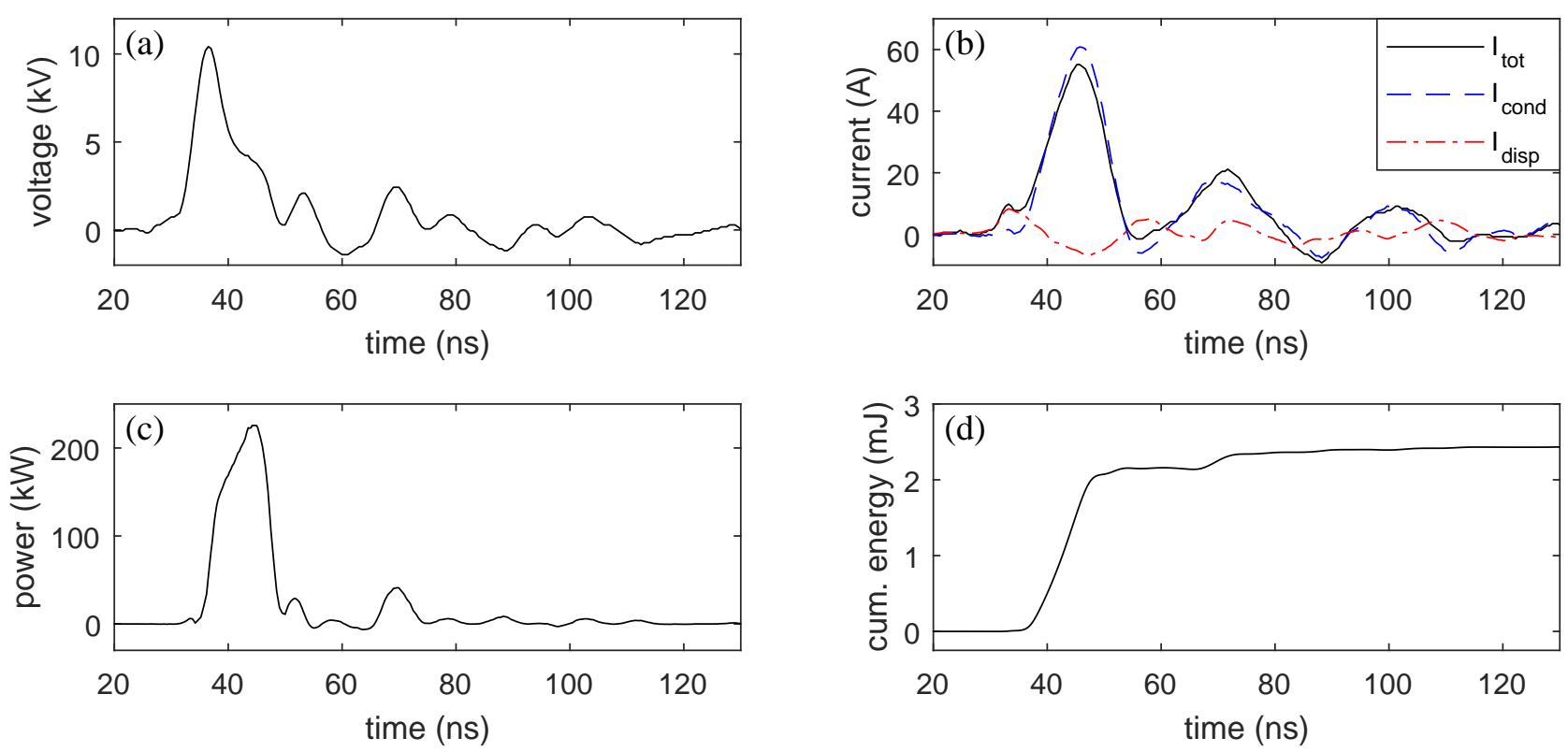

Figure 8: Representative signals of spark discharges: voltage $U$ (a); measured total current $I_{\text {tot }}$, conductive current $I_{\text {cond }}$, and displacement current $I_{\text {disp }}$ (b); power $P_{\text {cond }}$ calculated with $U \& I_{\text {cond }}$ (c); cumulative energy $E_{\text {cond }}(\mathrm{d})$

Table 1: Parameter combinations of gap distance, pulse repetition frequency, and reduced electric field for which acoustic and electrical energy measurements were made; modulation method is also given.

\begin{tabular}{lcccccccccccc|cc}
\hline Case & 1 & 2 & 3 & 4 & 5 & 6 & 7 & 8 & 9 & 10 & 11 & 12 & 13 & 14 \\
Modulation & BM & BM & BM & BM & BM & BM & BM & BM & BM & BM & BM & BM & PDM & PDM \\
\hline$d(\mathrm{~mm})$ & 4 & 4 & 4 & 4 & 4.5 & 4.5 & 4.5 & 4.5 & 5 & 5 & 5 & 5 & 4 & 4 \\
$f_{\text {PRF }}(\mathrm{kHz})$ & 20 & 25 & 27 & 29 & 20 & 25 & 27 & 29 & 20 & 25 & 27 & 29 & 30 & 30 \\
$E / N(\mathrm{Td})$ & 96 & 95 & 96 & 92 & 90 & 90 & 86 & 85 & 87 & 83 & 80 & 79 & 100 & 78 \\
\hline
\end{tabular}

\section{Results and discussion}

Spark formation and sound source amplitude were investigated for both modulation methods and for various gap distances, pulse repetition frequencies $f_{\mathrm{PRF}}$, and average reduced electric field $E / N$, see Tab. 1. In the present work, $E / N$ is calculated from the mean peak voltage. To ensure discharges in the spark regime, PDM signals were investigated with a maximum pulse repetition frequency of $30 \mathrm{kHz}$ and a gap distance of $4 \mathrm{~mm}$. PDM signals are generated with two different maximum pulse energy levels of approximately $2.2 \mathrm{~mJ}(E / N=100 \mathrm{Td})$ and $1.0 \mathrm{~mJ}(E / N=78 \mathrm{Td})$. For both methods, signals with modulation frequencies from 80 to $1000 \mathrm{~Hz}$ and duty cycles in the range of 0.05 to 0.95 were investigated. The mean energy of the discharges is between 1.0 and $2.5 \mathrm{~mJ}$, depending on the pulse repetition frequency, gap distance, and the curvature of the electrode tips.

Representative pressure signals and corresponding spectra, measured at microphone position $\mathrm{us}_{2}$ in the impedance tube (see Fig. 4), are shown in Fig. 9 for burst-modulated and in Fig. 10 for pulsedensity modulated signals. The top figures qualitatively illustrate the corresponding trigger signals. A clear response at the modulation frequency of $208 \mathrm{~Hz}$ with higher harmonics can be observed for both methods. Due to the square wave form, odd harmonics are more pronounced for burst signals.

\subsection{Energy per pulse and initialization pulses}

In order to determine the component of the electric power at the modulation frequency, it is essential to consider the development of the pulse energy during one modulation period. During burst- 

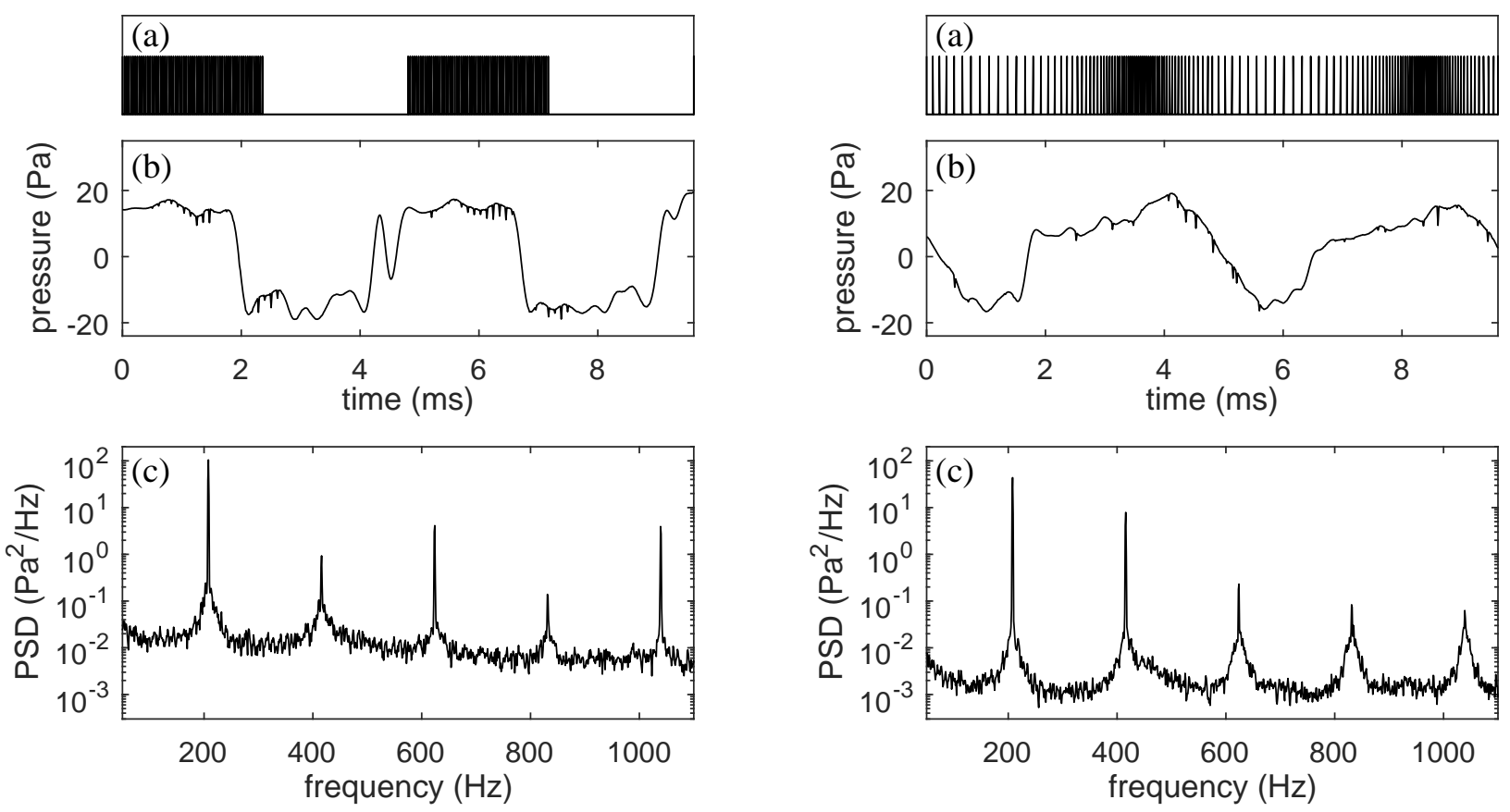

Figure 9: Burst modulation: (a) sketch of trigger signal, (b) pressure signal and (c) corresponding power spectral density, measured in the impedance tube (microphone position: $\mathrm{us}_{2}$, see Fig. 4); $f_{\mathrm{mod}}=208 \mathrm{~Hz}$, $f_{\mathrm{PRF}, \max }=28 \mathrm{kHz}, \mathrm{DC}=0.5$

Figure 10: Pulse-density modulation: (a) sketch of trigger signal, (b) pressure signal and (c) corresponding power spectral density, measured in the impedance tube (microphone position: us $_{2}$, see Fig. 4 ); $f_{\text {mod }}=$ $208 \mathrm{~Hz}, f_{\mathrm{PRF}, \max }=28 \mathrm{kHz}, \mathrm{DC}=0.5$

modulated plasma actuation, weak pulses without spark formation occur at the beginning of each burst-modulated pulse train. The pulse energy of these 2 to 5 pulses is very low compared to the following ones, independent of the duty cycle and electrode gap distance. The average electrical power at the modulation frequency is thus reduced, and it is important to consider this effect. To this end, four phase-averaged measurements of 40 pulses in the modulation cycle were acquired. For burst-modulated signals, the first 25 pulses and 15 pulses equidistantly distributed over the remaining high-gated period were measured. Figure 11 shows measured (open circles) and linearly interpolated (background color map) pulse energies, corresponding to burst-modulated plasma forcing. The energy is measured for duty cycles in the range of 0.05 to 0.95 . Top and bottom plots represent the corresponding pulse trains for maximum (top) and minimum (bottom) duty cycle. The pulse energy remains fairly constant for burst-modulated signals, except for the first few initialization pulses. This justifies using interpolation for determining the non-measured pulse energies.

Pai [36] observed an increasing pulse energy with the pulse repetition frequency in a pin-to-pin set-up at $1000 \mathrm{~K}$ with $1.6 \mathrm{~m} / \mathrm{s}$ flow speed. For pulse-density modulated signals, the distance between two pulses is not constant and, therefore, neither is the pulse energy. Pre-tests have shown that two phase-averaged measurements of 50 equidistant pulses are able to capture the fluctuating pulse energy, for signals investigated in this work. Values that were not measured are determined by interpolation. Figure 12 shows representative pulse energies for pulse-density modulated signals at a modulation frequency of $144 \mathrm{~Hz}$ (analogous to Fig. 11). The duty cycle for PDM signals describes the ratio of the actual number of pulses per period to the maximum number with a constant pulse repetition frequency of $30 \mathrm{kHz}$. Regions with a low momentary pulse repetition frequency (bottom left of the middle figure) contain pulses with lower energy than regions with a higher pulse repetition frequency (top right). Furthermore, the energy decreases slightly in regions with very high pulse repetition frequency (see marked region).

Figure 13 shows the measured pulse energy from pulse-density modulated signals of all investi- 


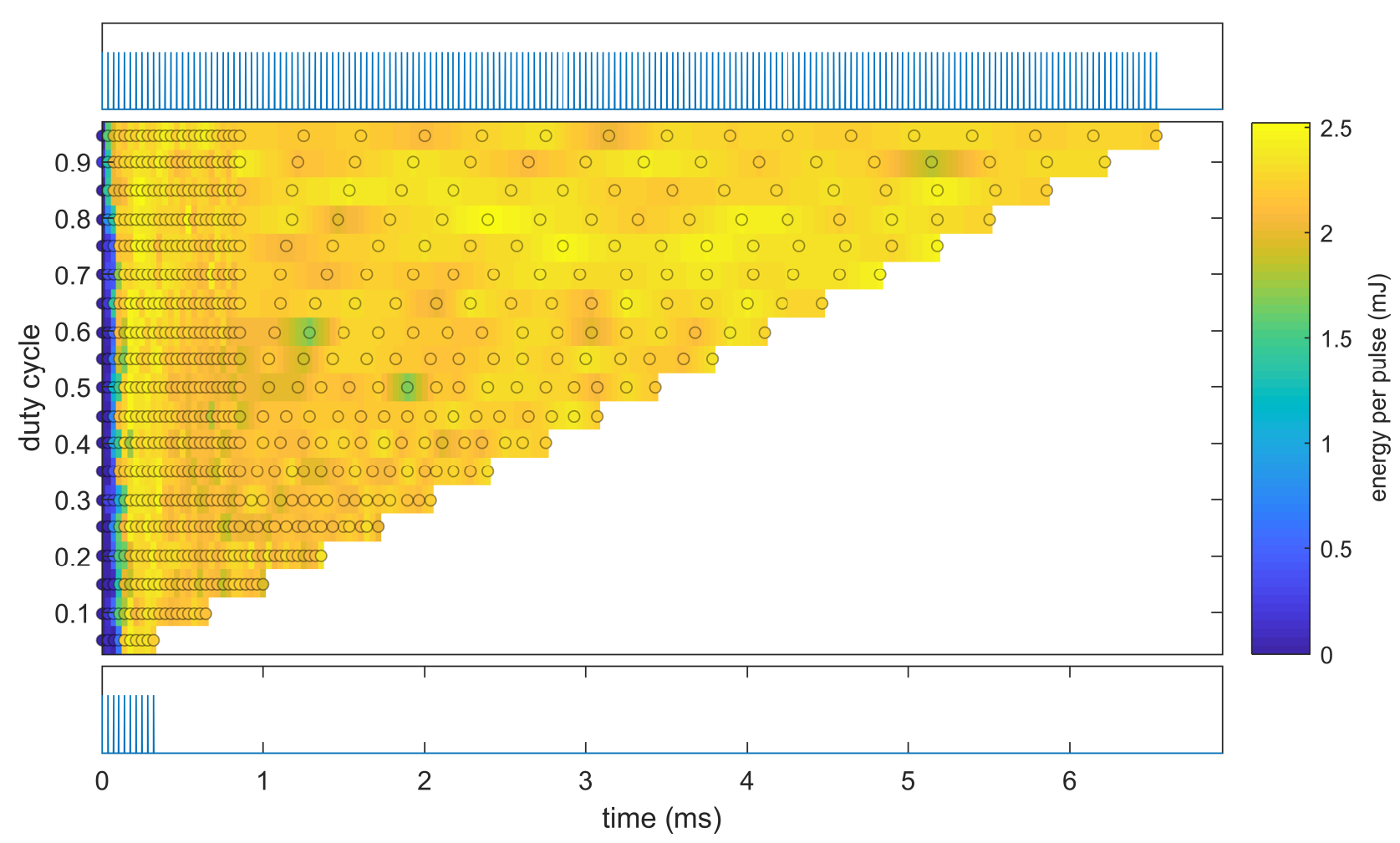

Figure 11: Pulse energy for burst-modulated high-frequency NRP discharge trains: measured (circles) and interpolated (colormap) pulse energy over one period (bottom axis) for various duty cycles at $f_{\text {mod }}=144 \mathrm{~Hz}$, $f_{\mathrm{PRF}}=28 \mathrm{kHz}$; top and bottom frames illustrate the pulse positions for the maximum duty cycle and for the minimum, respectively.

gated cases (see Tab. 1). The instantaneous pulse repetition frequency is defined by the time to the previous pulse. Although the pulse energy is not only affected by the previous pulse (e.g., gap distance, electrode geometry, remaining species [37]), a clear trend can be observed. The pulse energy increases with the pulse repetition frequency until the maximum is reached at approximately $10 \mathrm{kHz}$. For higher pulse repetition frequencies, the pulse energy slightly decreases. To remain within the spark regime, the minimum pulse repetition frequency in the measured test cases is set to $2 \mathrm{kHz}$.

\subsection{Effect of modulation duty cycle on sound amplitude and the advantage of the noise mitigation step}

The effect of the duty cycle on the amplitude of the acoustic source is determined from measurements with burst and pulse-density modulated signals, for different modulation frequencies. The duty cycle is varied by changing the number of pulses per modulation period. To illustrate the advantage of the noise mitigation technique, the data are evaluated with and without the additional processing step described in Section 4.2. This aspect is discussed first.

\section{Advantage of the noise mitigation technique}

Due to the steep rising edge and the high amplitude of the pulse voltage (see Fig. 8), a strong electromagnetic field, correlated with the modulation signal, contaminates the microphone signals. The technique described in Section 4.2 is applied to remove these spurious components. Figure 14 shows the measured source amplitude with and without noise mitigation for different duty cycles and modu- 


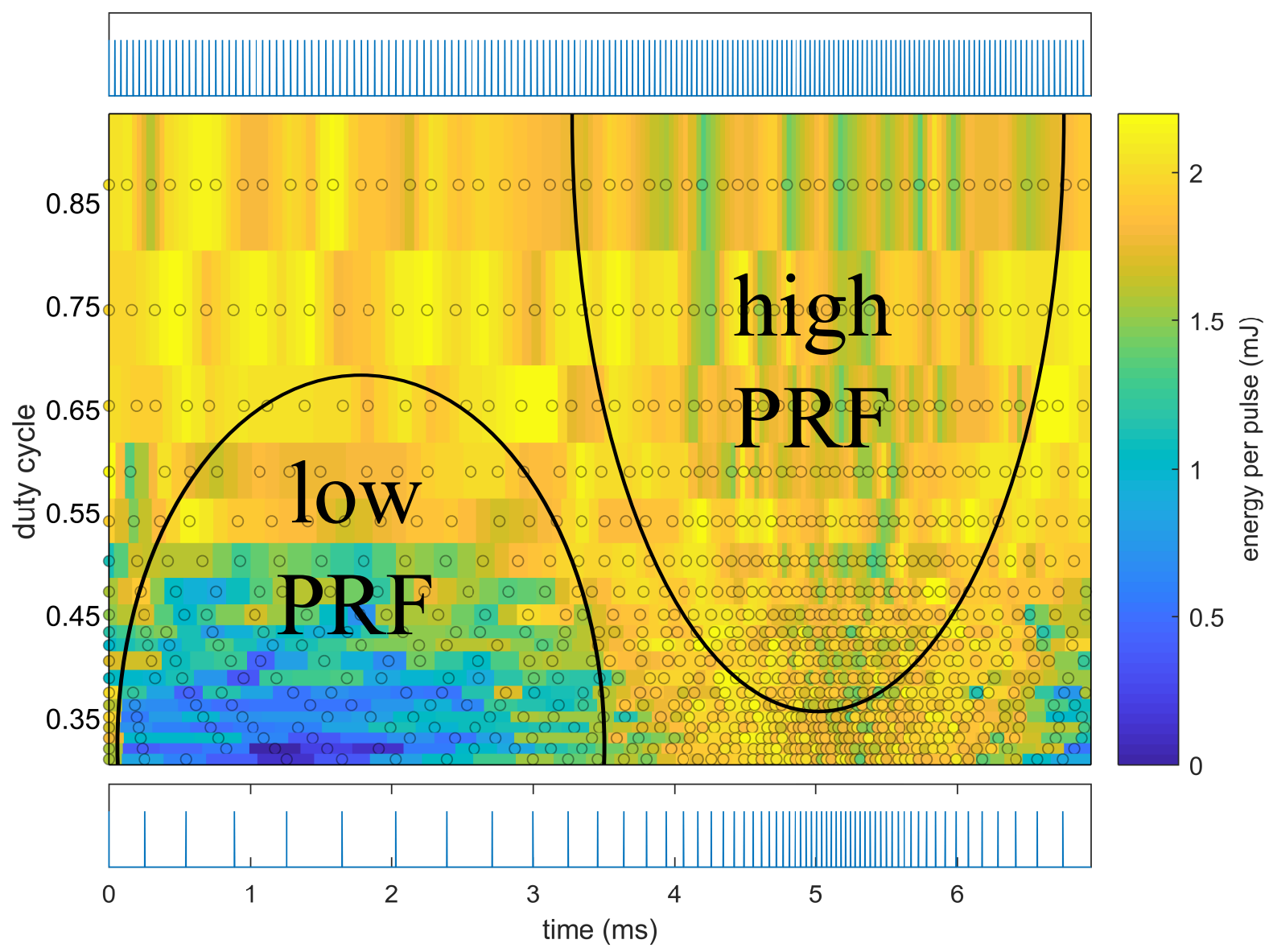

Figure 12: Pulse energy for pulse-density modulated high-frequency NRP discharge trains: measured (circles) and interpolated (colormap) pulse energy over one period (bottom axis) for various duty cycles at $f_{\bmod }=$ $144 \mathrm{~Hz}, f_{\mathrm{PRF}, \max }=30 \mathrm{kHz}$; top and bottom frames illustrate the pulse positions for the maximum duty cycle and for the minimum, respectively.

lation frequencies. The dashed line shows the amplitude when a constant pulse energy is assumed (see also Fig. 3), multiplied by the maximum value to fit the data obtained with noise mitigation. Without noise mitigation, the data at low modulation frequencies is significantly contaminated by spurious components. Data calculated with noise mitigation according to Eq. 19 shows the expected duty cycle dependence. In the following sections, only results from noise-removed data are discussed.

The sensitivity of the multi-microphone method to measurement errors is described by Jang et al. [38]. They show that the estimation error is proportional to a singularity factor, which is calculated from the microphone locations as well as the number of microphones used. Figure 15 shows representative singularity factors dependent on the microphone number in the upstream and downstream section of the impedance tube for the frequency range of 40 to $1200 \mathrm{~Hz}$ (see Fig. 4). An increasing number of microphones reduces the singularity factor significantly; therefore, the results shown in this work are calculated with five microphones. However, below $150 \mathrm{~Hz}$ the singularity factor strongly increases, which can be also observed in the frequency dependent level of spurious components in Fig. 14.

\section{Effect of modulation duty cycle on sound amplitude}

Figure 16 compares the duty-cycle dependence of the measured source amplitudes for burst [Fig. 16(a)] and pulse-density modulated [Fig. 16(b)] signals with the corresponding ideal curve (dashed). For burst-modulated signals, the duty-cycle dependence of the measured source amplitudes is proportional to $\sin (\pi \mathrm{DC})$. The measured source amplitudes are slightly shifted to higher duty cycles. This originates from the few initialization pulses (non-ignited sparks, see Sec. 5.1) at the start of each new 


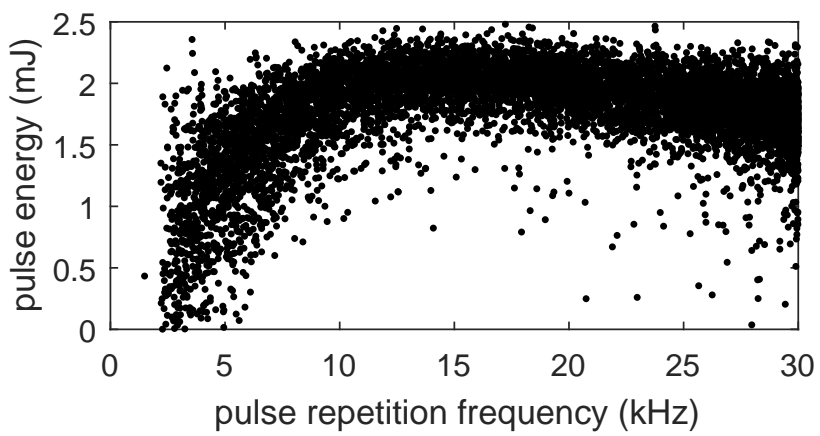

Figure 13: Pulse energy as a function of the pulse repetition frequency, measured from pulse-density modulated signals: gap distance $4 \mathrm{~mm}, f_{\mathrm{PRF}, \max }=30 \mathrm{kHz}$

modulation period. The relative contribution of the non-ignited sparks increases for higher modulation frequencies because the total number of pulses per period decreases. This explains the stronger shift for higher modulation frequencies, which is observed in Fig. 16(a).

The effect of low-energy pulses becomes negligibly small for pulse-density modulated signals. Due to the characteristics of the modulation method, the number of low-energy pulses decreases with increasing duty cycle and becomes zero for signals with duty cycle larger than $50 \%$ (see Fig. 12). Furthermore, due to the shorter pulse distances, the impact of low-energy pulses is generally smaller. Hence, the measured acoustic source amplitude is very close to the ideal one [Fig. 16(b)].

\subsection{Comparison of the measured source amplitudes with the theoretical estimate}

If all the electrical power at the modulation frequency is contributed to unsteady heating, over a timescale that is short compared to the acoustic period, an analytic estimate of the acoustic source amplitude is given by Eq. (8), with the heat release rate fluctuation $\hat{Q}$ replaced by the electric power amplitude [Eq. (12)]. Figure 17 shows the measured data and those calculated from the pulse energy for burst-modulated [Fig. 17(a)] and pulse-density-modulated [Fig. 17(b)] signals for all test cases (see Tab. 1). Taking into account measurement inaccuracies in the experimental acoustic source determination as well as measurement errors in the pulse energy, experimental data and model agree well. For burst-modulated signals, a direct dependence of the source amplitude on gap distance, pulse repetition frequency, average pulse energy, and reduced electric field could not be observed. These parameters affect the sound source amplitude indirectly through the pulse energy and the electric power component at the modulation frequency [Eq. (12)].

The accuracy of the experimentally determined wave amplitudes can be estimated by comparing the measured pressure phasors, $\hat{\mathbf{p}}_{\text {meas }}$, with those reconstructed from the wave amplitudes by Eq. 14 . The latter is referred to as the estimated pressure field, $\hat{\mathbf{p}}_{\text {est }}$. The relative error estimate is then obtained as

$$
\varepsilon=\frac{\left|\hat{\mathbf{p}}_{\text {meas }}-\hat{\mathbf{p}}_{\text {est }}\right|}{\left|\hat{\mathbf{p}}_{\text {meas }}\right|}
$$

This relative error is used for the vertical error bars in Fig. 17. Horizontal error bars correspond to uncertainty in the calculated source amplitude originating from the measurement inaccuracy of the pulse energy. The latter is estimated from pulses without discharge, for which the pulse energy should be zero when calculated by the conductive current only.

\section{Conclusions}

Sound generation from low-frequency modulated NRP discharges was investigated experimentally. Acoustic waves with amplitudes up to $40 \mathrm{~Pa}$ could be generated with burst and pulse-density modula- 

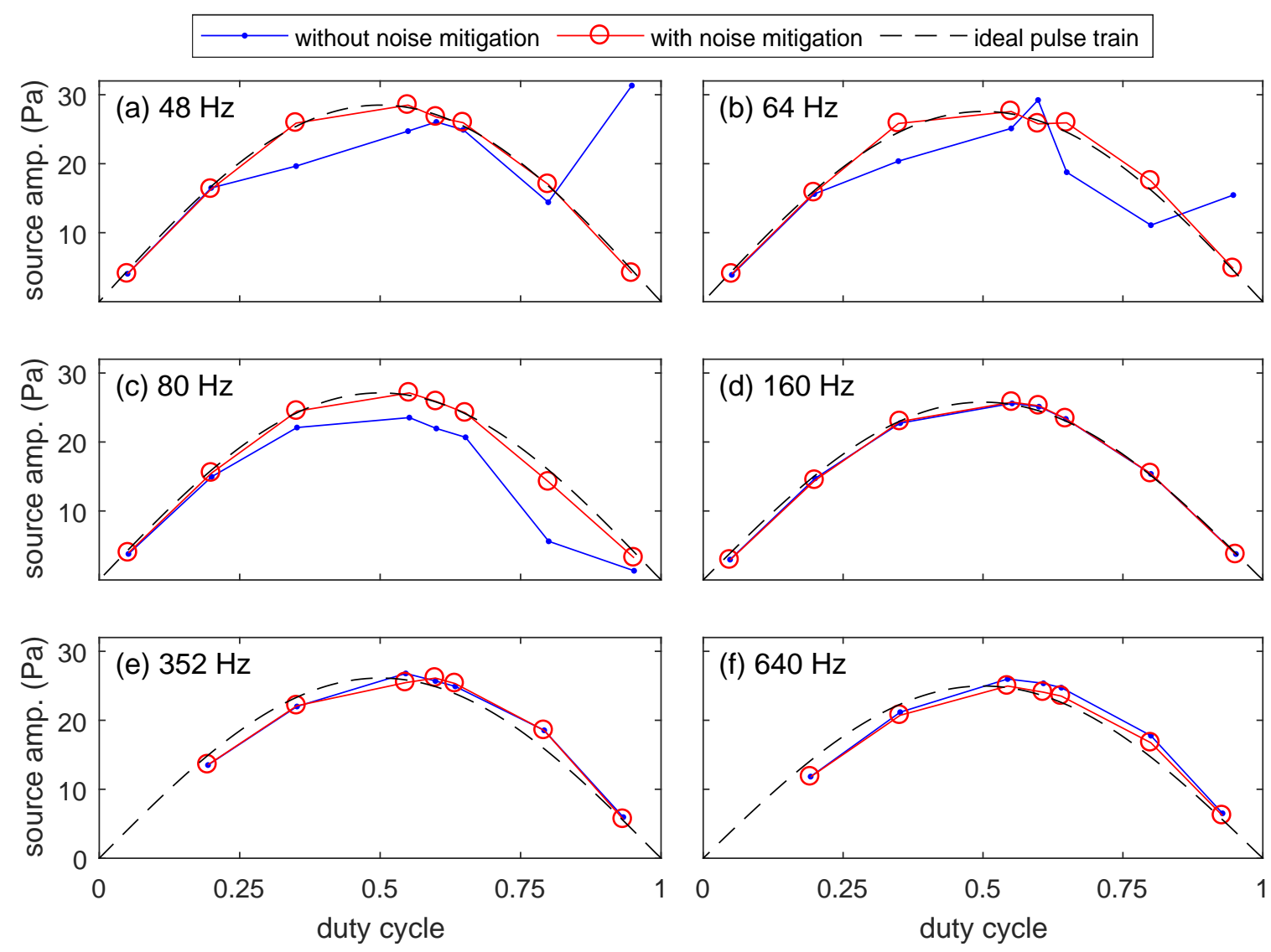

Figure 14: Acoustic source amplitude for various duty cycles measured at different modulation frequencies with burst modulation: comparison of results with and without noise mitigation.

tion, in the frequency range of 50 to $1000 \mathrm{~Hz}$, which is much lower than the pulse repetition frequency (10 to $30 \mathrm{kHz}$ ). The spectral amplitude at the modulation frequency can be varied with both methods by controlling the number and/or temporal position of the discharges in one period.

The measured pressure signals were strongly contaminated by EM disturbances associated with the nanosecond discharges. The impact of signal contamination on the measured acoustic source amplitudes is particularly high at low modulation frequencies because the identification of the acoustic wave amplitudes is more sensitive at larger wavelengths. Spurious components were removed from the microphone signals with a special technique. This technique utilizes the acoustic boundary conditions upstream and downstream of the measurement section, which can be measured with high accuracy without plasma discharges.

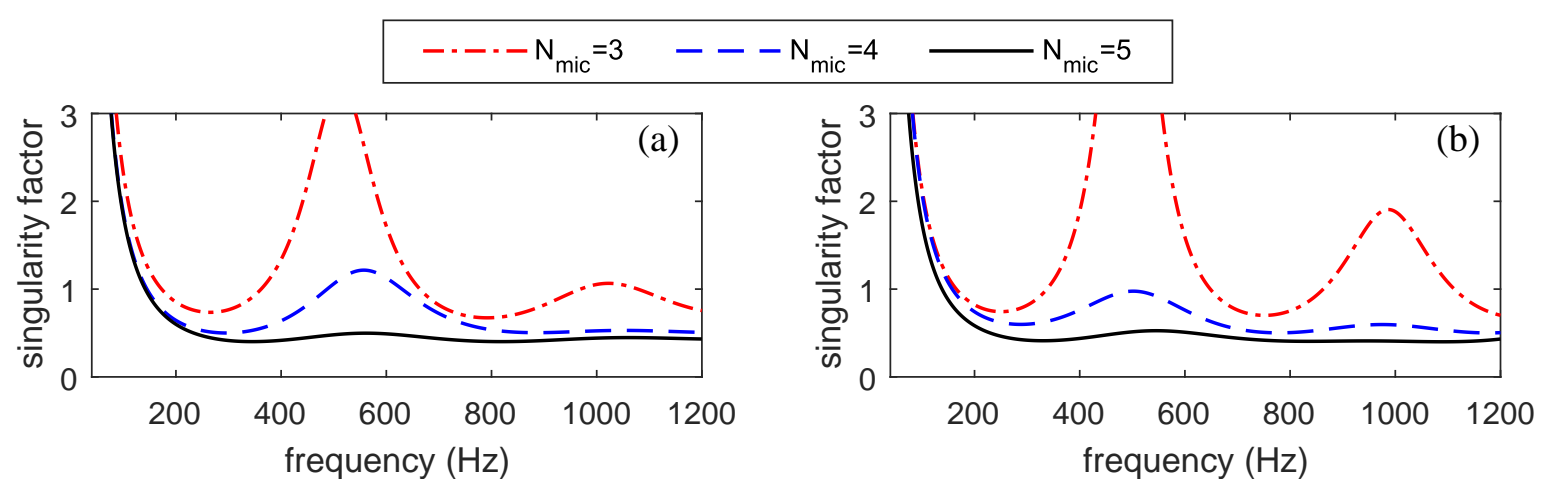

Figure 15: Singularity factors SF associated with the (a) upstream and (b) downstream microphone array, for different numbers of microphones. 


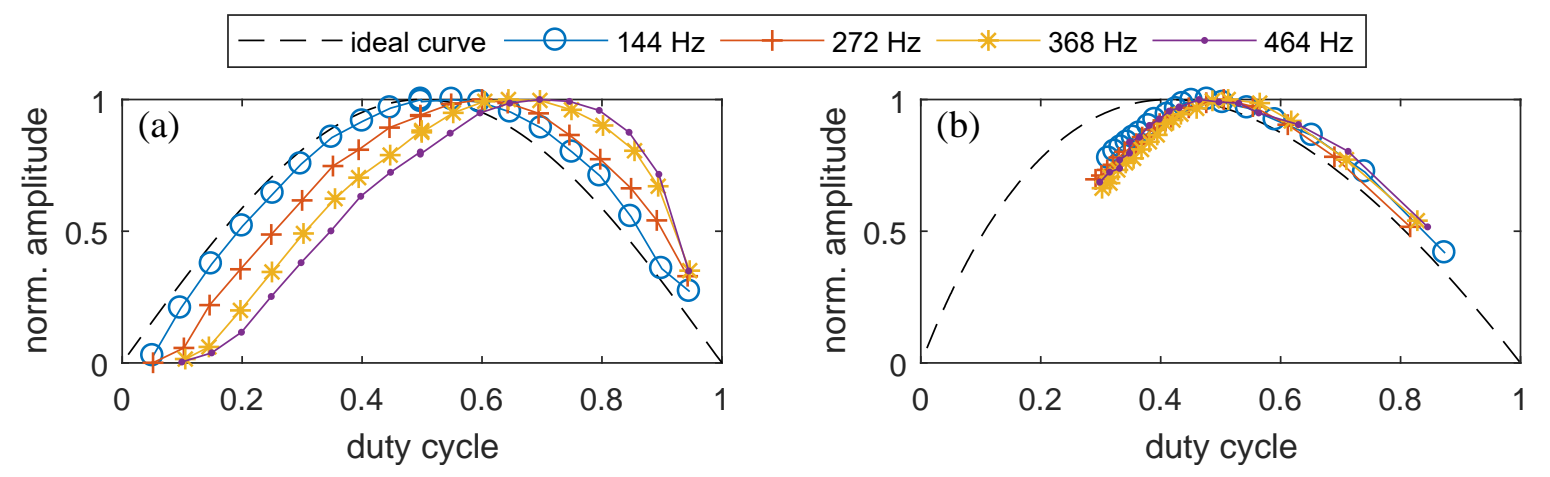

Figure 16: Effect of duty cycle on the sound source amplitude for four modulation frequencies. $f_{\mathrm{PRF}}=28 \mathrm{kHz}$, $d=4 \mathrm{~mm}$; (a) burst-modulated signals; (b) pulse-density-modulated signals
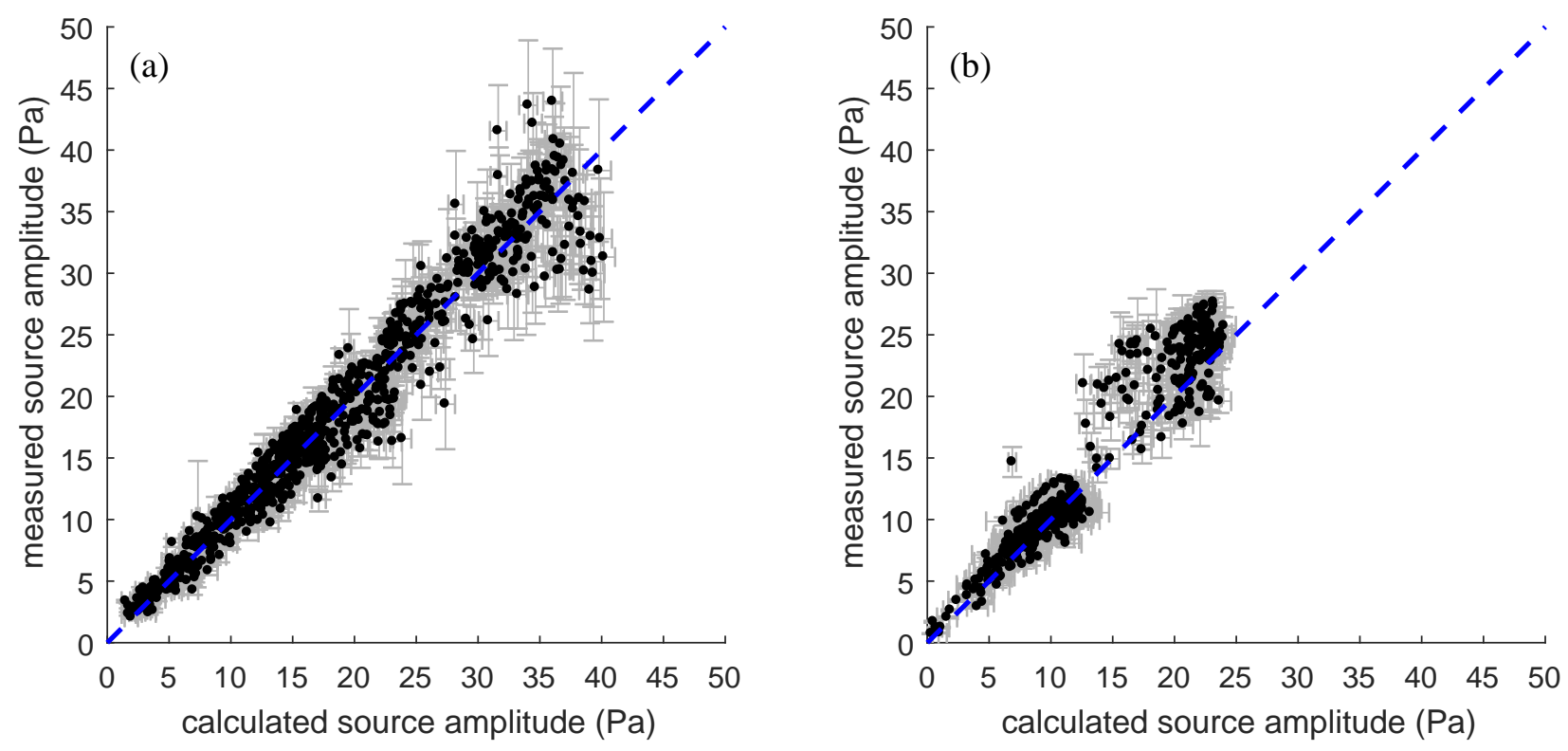

Figure 17: Comparison of measured sound source amplitudes and calculations using Eq. (8), with the heat release rate fluctuation amplitude replaced by the electrical power amplitude at the modulation frequency. (a) Burst-modulated signals, (b) pulse-density-modulated signals; ideal correspondence is given by the dashed blue line.

The sound generation from low-frequency modulated NRP discharges can be estimated with good quantitative accuracy with a model based on unsteady heating. The only input to this model is the Fourier component of the electrical power at the modulation frequency. This quantity can be calculated from the energy per pulse and the pulse sequence.

When burst modulation is applied, no breakdown occurs for the first 2 to 5 high-voltage pulses in every modulation period. This is observed in many repeated energy measurements but becomes also apparent when the duty-cycle dependence of the sound source amplitude is considered, which features a maximum shifted to duty cycles higher than 50\%. As the number of non-fired sparks is approximately constant, this effect is more pronounced at higher modulation frequencies, for which the total number of pulses per modulation period decreases (when the PRF is held constant).

Pulse-density modulated pulse trains with a duty cycle below $50 \%$ are found to be overestimated compared to the model predicted ideal case, while sequences with a higher duty cycle matched well for all tested cases. Non-ignited sparks occur only when the inter-pulse time is larger than $0.1 \mathrm{~ms}$. Thus, pulse-density modulation is not affected by non-ignited pulses when the duty cycle is at least $50 \%$. However, for a given minimum pulse distance (maximum PRF), burst modulation provides a larger Fourier component at the modulation frequency compared to pulse-density modulation and, therefore, a higher sound source amplitude. 
Over the entire set of experimental data, the measured sound source amplitude agreed well with the theoretical model based on unsteady heating. The effects of pulse repetition frequency, gap distance, reduced electric field, and modulation method are captured through the electrical pulse energy. We can thus conclude that for the present investigations with NRP spark discharges corresponding to reduced electric fields in the range of $78 \mathrm{Td}$ to $100 \mathrm{Td}$, cumulative unsteady heating is the dominant source of sound at modulation frequencies up to $1 \mathrm{kHz}$.

\section{Acknowledgements}

This work was supported by the Agence Nationale de la Recherche (ANR) and the German Research Foundation (DFG) through the DRACO project (grant numbers ANR-13-IS09-0004, MO 2551/1)

\section{References}

[1] Duddell, W. D. B. "Some Experiments on the Direct-Current Arc". In: Nature 63 (1900), pp. 182-183.

[2] Mazzola, M. S. and Molen, G. "Modeling of a dc glow plasma loudspeaker". In: The Journal of the Acoustical Society of America 81.6 (1987), pp. 1972-1978.

[3] Babcock, W. R., Baker, K. L., and Cattaneo, A. G. "Musical Flames". In: Nature 216.5116 (1967), pp. 676-678.

[4] Küchler, A. Hochspannungstechnik. 2009.

[5] Bastien, F. “Acoustics and gas discharges: applications to loudspeakers". In: Journal of Physics D: Applied Physics 20.12 (1987), pp. 1547-1557.

[6] Raizer, Y. P. Gas discharge physics. Springer-Verlag, 1991.

[7] Sutton, Y. "Electro-acoustic coupling in a plasma gas". PhD thesis. The Open University, 2011.

[8] Aleksandrov, N. L., Kindysheva, S. V., Nudnova, M. M., and Starikovskiy, A. Y. "Mechanism of ultra-fast heating in a non-equilibrium weakly ionized air discharge plasma in high electric fields". In: Journal of Physics D: Applied Physics 43 (2010), 255201 (19pp).

[9] Rusterholtz, D. "Nanosecond Repetitively Pulsed Discharges in Atmospheric Pressure Air". $\mathrm{PhD}$ thesis. Ecole Centrale Paris, 2014.

[10] Levchenko, I., Ostrikov, K., and Mariotti, D. "The production of self-organized carbon connections between Ag nanoparticles using atmospheric microplasma synthesis". In: Carbon 47.1 (2009), pp. 344-347.

[11] Boeuf, J., Lagmich, Y., Calligari, T., and Pitchford, L. "Electrohydrodynamic Force and Acceleration in Surfaces Discharges". In: 37th AIAA Plasmadynamics and Lasers Conference. Reston, Virigina: American Institute of Aeronautics and Astronautics, 2006.

[12] Bérard, P., Lacoste, D., and Laux, C. "Measurements and Simulations of the Ionic wind Produced by a DC Corona Discharge in Air, Helium and Argon". In: 38th Plasmadynamics and Lasers Conference. Fluid Dynamics and Co-located Conferences. Reston, Virigina: American Institute of Aeronautics and Astronautics, 2007.

[13] Elias, P.-Q., Chanetz, B., Larigaldie, S., and Packan, D. "Study of the Effect of Glow Discharges Near a M = 3 Bow Shock”. In: AIAA Journal 45.9 (2007), pp. 2237-2245.

[14] Kim, W., Snyder, J., and Cohen, J. "Plasma assisted combustor dynamics control". In: Proceedings of the Combustion Institute 35.3 (2015), pp. 3479-3486. 
[15] Moeck, J., Lacoste, D., Laux, C., and Paschereit, C. "Control of combustion dynamics in a swirl-stabilized combustor with nanosecond repetitively pulsed discharges". In: 51st AIAA Aerospace Sciences Meeting including the New Horizons Forum and Aerospace Exposition. Reston, Virigina: American Institute of Aeronautics and Astronautics, 2013.

[16] Lacoste, D. A., Moeck, J. P., Durox, D., Laux, C. O., and Schuller, T. "Effect of Nanosecond Repetitively Pulsed Discharges on the Dynamics of a Swirl-Stabilized Lean Premixed Flame". In: Journal of Engineering for Gas Turbines and Power 135.10 (2013), p. 101501.

[17] Pilla, G., Galley, D., Lacoste, D. A., Lacas, F., Veynante, D., and Laux, C. O. "Stabilization of a Turbulent Premixed Flame Using a Nanosecond Repetitively Pulsed Plasma". In: IEEE Transactions on Plasma Science 34.6 (2006), pp. 2471-2477.

[18] Ju, Y. and Sun, W. "Plasma assisted combustion: Dynamics and chemistry". In: Progress in Energy and Combustion Science 48 (2015), pp. 21-83.

[19] Rusterholtz, D. "Nanosecond Repetitively Pulsed Discharges in Atmospheric Pressure Air". In: Ph.D. Thesis, École Centrale Paris (2012), p. 220.

[20] Dowling, A. P. and Mahmoudi, Y. "Combustion noise". In: Proceedings of the Combustion Institute 35.1 (2015), pp. 65-100.

[21] Truffaut, J.-M., Searby, G., and Boyer, L. "Sound emission by non-isomolar combustion at low Mach numbers". In: Combustion Theory and Modelling 2.4 (1998), pp. 423-428.

[22] Xu, D. A., Lacoste, D. A., Rusterholtz, D. L., Elias, P.-Q., Stancu, G. D., and Laux, C. O. "Experimental study of the hydrodynamic expansion following a nanosecond repetitively pulsed discharge in air”. In: Applied Physics Letters 99.12 (2011), p. 121502.

[23] Xu, D. A., Shneider, M. N., Lacoste, D. A., and Laux, C. O. "Thermal and hydrodynamic effects of nanosecond discharges in atmospheric pressure air". In: Journal of Physics D: Applied Physics 47.23 (2014), p. 235202.

[24] Bölke, O., Moeck, J. P., and Lacoste, D. A. "Sound generation and control of thermoacoustic instabilities by nanosecond plasma discharges". In: Proceedings of the 23rd International Congress on Sound and Vibration. July 10-14, Athens, Greece, 2016.

[25] Lavrentjev, J., Åbom, M., and Bodén, H. "A measurement method for determining the source data of acoustic two-port sources". In: Journal of Sound and Vibration 183.3 (1995), pp. $517-$ 531.

[26] Fickett, W. and Davis, W. C. Detonation - Theory and Experiment. Dover Publications, INC., 1979.

[27] Hirschberg, A. and Rienstra, S. An Introduction to Acoustics. IWDE-Report. 2014.

[28] Lo, A., Cessou, A., and Vervisch, P. "Space and time analysis of the nanosecond scale discharges in atmospheric pressure air: II. Energy transfers during the post-discharge". In: Journal of Physics D: Applied Physics 47.11 (2014), p. 115202.

[29] Rusterholtz, D. L., Lacoste, D. A., Stancu, G. D., Pai, D. Z., and Laux, C. O. "Ultrafast heating and oxygen dissociation in atmospheric pressure air by nanosecond repetitively pulsed discharges". In: Journal of Physics D: Applied Physics 46.46 (2013), p. 464010.

[30] Lo, A., Cessou, A., Boubert, P., and Vervisch, P. "Space and time analysis of the nanosecond scale discharges in atmospheric pressure air: I. Gas temperature and vibrational distribution function of N2 and O2”. In: Journal of Physics D: Applied Physics 47.11 (2014), p. 115201.

[31] Znamenskaya, I. A., Latfullin, D. F., Lutskiy, A. E., Mursenkova, I. V., and Sysoev, N. N. "Development of hydrodynamic disturbances from zone of distributed surface sliding discharge". In: Zhurnal Tekhnicheskoi Fizik 77 (2007). 
[32] Popov, N. A. "Investigation of the mechanism for rapid heating of nitrogen and air in gas discharges”. In: Plasma Physics Reports 27.10 (2001), pp. 886-896.

[33] Xu, D. "Thermal and Hydrodynamic Effects of Nanosecond Discharges in Air and Application to Plasma-Assisted Combustion". PhD thesis. Ecole Centrale Paris, 2013.

[34] Pierce, A. D. Acoustics: An Introduction to Its Physical Principles and Applications. Acoustical Society of America, 1994.

[35] Åbom, M. “A note on the experimental determination of acoustical two-port matrices". In: Journal of Sound and Vibration 155.1 (1992), pp. 185-188.

[36] Pai, D. "Nanosecond Repetitively Pulsed Plasmas in Preheated Air at Atmospheric Pressure". $\mathrm{PhD}$ thesis. Ecole Centrale Paris.

[37] Heitz, S. A., Moeck, J. P., Schuller, T., Veynante, D., and Lacoste, D. A. "Influence of air flow parameters on nanosecond repetitively pulsed discharges in a pin-annular electrode configuration”. In: Journal of Physics D: Applied Physics 49.15 (2016), p. 155205.

[38] Jang, S.-H. and Ih, J.-G. "On the multiple microphone method for measuring in-duct acoustic properties in the presence of mean flow". In: The Journal of the Acoustical Society of America 103.3 (1998), pp. 1520-1526. 